Check for updates

London, UK

Cite this as: BMJ 2020;370:m2935 http://dx.doi.org/10.1136/bmj.m2935 Published: 22 July 2020

\section{Intensive immunosuppression reduces deaths in covid-19-associated cytokine storm syndrome, study finds}

Susan Mayor

Intensive immunosuppressive treatment with high dose glucocorticoids, followed by an interleukin-6 (IL-6) receptor antagonist where needed, accelerates respiratory recovery and reduces deaths in patients with covid-19-associated cytokine storm syndrome (CSS), an observational study ${ }^{1}$ has shown.

Researchers from the Netherlands conducted a historically controlled comparison of a strategy with intensive immunosuppression and close monitoring versus supportive care.

"This comparison suggests that clinically relevant improvement of respiratory status was $79 \%$ more likely and can be accelerated by a median of seven days. Hospital mortality can be reduced by $65 \%$ and the need for mechanical ventilation by $71 \%$," the team, led by Sofia Ramiro, from Zuyderland Medical Center in Heerlen, reported.

They added, "These outcomes were robust, and, especially if confirmed in randomised trials, highly relevant from a medical and societal perspective. Glucocorticoids are safe, widely available, and inexpensive."

Covid-19-associated CSS is an important complication of SARS-CoV-2 infection. It occurs in $10-20 \%$ of patients with covid-19 pneumonia and is associated with significant mortality.

From 1 April 2020 the researchers treated all patients with covid-19-associated CSS admitted to Zuyderland Medical Center with high dose intravenous methylprednisolone for five consecutive days. The IL-6 receptor blocker tocilizumab was added in those showing insufficient improvement.

A total of 86 patients were included in the study, with covid-19-associated CSS being defined as rapid respiratory deterioration plus at least two of three raised biomarkers (C-reactive protein >10omg/L; ferritin $>900 \mu \mathrm{g} / \mathrm{L}$; D-dimer $>1500 \mu \mathrm{g} / \mathrm{L}$ ). Each of these patients was compared with a matched control treated with supportive care between 7 and 31 March.

Results, published in Annals of Rheumatic Diseases, showed that patients treated with intensive immunosuppression were $79 \%$ more likely to show respiratory improvement than controls (hazard ratio 1.8, $95 \%$ confidence interval 1.2 to $2.7, \mathrm{P}=0.0025$ ). This was defined as $>2$ stages of improvement on a seven item World Health Organization endorsed scale for trials in patients with severe influenza pneumonia, or hospital discharge.

Hospital mortality was 65\% lower with intensive immunosuppressive therapy (HR 0.35, 95\% CI 0.19 to 0.65 , $\mathrm{P}=0.0004)$. And the likelihood of requiring invasive mechanical ventilation was reduced by $71 \%$ compared with patients receiving supportive care only (HR 0.29; 95\% CI 0.14 to $0.65, \mathrm{P}=0.0003$ ).

The treatment effects remained after excluding the $43 \%$ of patients who also received tocilizumab. The researchers said this suggested that a "clinically relevant treatment effect can be reached by high dose glucocorticoid alone."

The researchers warned that their study, which received no research funding, cannot be "interpreted as a randomised controlled trial." But they said, "While we, in principle, advocate further study strategies like ours in randomised controlled trials, it is an interesting philosophical question whether randomised controlled trials with 'supportive care only' in the control group will be justifiable in future.”

Ramiro said, “A strong point of our study has been the meticulous selection of those patients more likely to benefit from immunosuppressive treatment, namely patients with a CSS. Other studies so far have in general included all admitted covid-19 patients."

The UK Randomised Evaluation of covid-19 therapy (RECOVERY) trial, which is comparing a range of possible treatments with usual care in patients hospitalised with covid-19, has also shown benefits with steroids. A preliminary report ${ }^{2}$ found that dexamethasone reduced deaths by a third in patients receiving invasive mechanical ventilation $(29.0 \% v 40.7 \%$, rate ratio $0.65,95 \% \mathrm{CI} 0.51$ to $0.82, \mathrm{P}<0.001)$, and by one fifth in patients receiving oxygen without invasive mechanical ventilation $(21.5 \%$ v $25.0 \%$, RR $0.80,95 \% \mathrm{CI} 0.70$ to 0.92, $\mathrm{P}=0.002$ ).

"The studies complement each other and it is reassuring that conclusions are similar. Still, the effect of our immunosuppressive treatment was much higher than seen in the RECOVERY trial," commented Ramiro. She suggested this was likely to be because of the selection of patients. "The RECOVERY trial included all patients admitted with covid-19. For our study, we carefully selected patients with covid-19-associated CSS as these are the patients that seem to have more benefit from immunosuppressive treatment. This has also been shown in our study," she said. 
"Recognising patients with covid-19-associated CSS is very relevant since these patients have a worse prognosis and a high mortality rate. We now have evidence that treatment suppressing the immune system helps these patients fighting against the disease,” Ramiro concluded.

\section{Correction: We have amended this article to correct the spelling of two authors' names (Sofia Ramiro and RLM Mostard).}

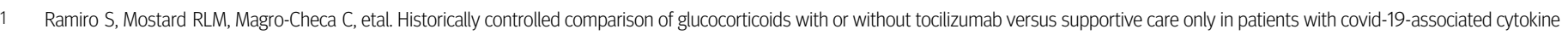
storm syndrome: results of the CHIC study. Ann Rheum Dis 2020;0:1-9doi: 10.1136/annrheumdis-2020-218479.

2 Effect of dexamethasone in hospitalized patients with covid-19: preliminary report. 22 June 2020 [Preprint] www.medrxiv.org/content/10.1101/2020.06.22.20137273v1.full.pdf

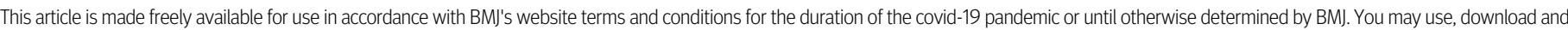
print the article for any lawful, non-commercial purpose (including text and data mining) provided that all copyright notices and trade marks are retained. 